\title{
El tratamiento de las locuciones en los manuales de ELE de nivel avanzado (B2)
}

\author{
The treatment of idioms in the Spanish as a foreign language \\ teaching textbooks for advanced Level (B2)
}

\author{
HAIYANG ZHU \\ UNIVERSIDAD AUTÓNOMA DE BARCELONA \\ haiyang.zhu@e-campus.uab.cat
}

\begin{abstract}
Resumen:
Las locuciones son unidades léxicas complejas que se caracterizan por su alto grado de idiomaticidad y su inmutabilidad formal, razón por la cual para los estudiantes de lengua extranjera (LE), comprender y aprender estas expresiones estereotipadas no es una tarea fácil, por lo tanto, se suelen quedar en un segundo plano en la clase de LE. En el presente artículo, tenemos como objetivo investigar el tratamiento que reciben las locuciones en los manuales de ELE más recientes con el fin de revelar las deficiencias que existen.
\end{abstract}

Palabras claves: locuciones, ELE, manuales de ELE.

\footnotetext{
Abstract:

The idioms are complex lexical units characterized by their high degree of idiomaticity and formal immutability, which is why for foreign language students, understanding and learning these stereotyped expressions is not an easy task, and therefore, they are usually left in the background in foreign language classes. In this article, we aim to investigate the treatment of idioms in the most recent Spanish as a foreign language teaching textbooks in order to reveal the deficiencies that exist.

Keywords: idioms, Spanish as a foreign language, teaching textbooks.
} 


\section{Introducción}

Las locuciones se suelen quedar en un segundo plano en las clases de ELE o tratarse simplemente como un fenómeno anecdótico (Cooper, 1998: 259). Sin embargo, en los últimos años, se han publicado numerosos estudios en el ámbito de la fraseología y, en concreto, sobre las locuciones ${ }^{1}$ así como gran número de trabajos sobre su didáctica ${ }^{2}$. Estos estudios argumentan que las unidades locucionales son un elemento relevante en la adquisición de ELE y hay que enseñarlas de una forma exhaustiva y sistemática por sus grandes valores comunicativos y culturales.

A partir de la afirmación de Cooper arriba mencionada, cabría preguntarse, hoy en día, si los nuevos estudios teóricos y las propuestas didácticas han ayudado a introducir mejoras en la enseñanza-aprendizaje de locuciones en clase de español como lengua extranjera (ELE) y qué problemas o deficiencias todavía existen. Además, es interesante también estudiar qué porcentaje ocupan las locuciones, cómo se enseñan estas expresiones estereotipadas y en qué grado se han aplicado las teorías pedagógicas más recientes. Con el objetivo de contestar estas preguntas, en el presente artículo hemos propuesto, como corpus de nuestro estudio, analizar cuatro manuales recientes de ELE de nivel avanzado (B2): Abanico B2, Aula Internacional 4 (Nueva Edición), Embarque 4 y Nuevo Sueña 3, en los cuales según las directrices del Marco Común Europeo de Referencia para las Lenguas (el MCER), las locuciones deberían recibir una mayor atención. Estos cuatro manuales se han publicado en las principales editoriales de ELE y disfrutan de popularidad tanto en España como en el campo de enseñanza de ELE de todo el mundo y, por tanto, son cuatro libros de texto de los más utilizados actualmente.

En el presente artículo, comenzamos por presentar una visión general de la locución y abordar su relevancia en la enseñanza de ELE. A continuación, tratamos de repasar y sintetizar las principales teorías y propuestas didácticas de esta unidad léxica para que seguidamente, examinemos su aplicación en los manuales de ELE. Después, presentamos el corpus de estudio y la metodología del trabajo. Posteriormente, analizamos el corpus y discutimos los resultados obtenidos. Y, por último, presentamos las conclusiones del trabajo y exponemos los problemas de la enseñanza de locuciones que existen en los manuales más recientes.

\footnotetext{
1 Se trata de los trabajos de Casares Sánchez (1992), Corpas Pastor (2006), Martí Sánchez (2012), Penadés Martínez (2012b), etc.

2 En el ámbito de enseñanza-aprendizaje de las locuciones, se destacan los estudios de García Muñíais (1997), Cooper (1998), Penadés Martínez (2004), Ettinger (2008), entre muchos otros.
} 


\section{Las locuciones}

La locución es "una combinación fija de palabras que funciona como elemento de la oración y cuyo significado no se corresponde con la suma de los significados de sus componentes" (Penadés Martínez, 2012a: 23). A partir de esta definición, se pueden sintetizar sus características principales. En primer lugar, la locución se considera una unidad léxica compuesta y se caracteriza por la polilexicalidad (Corpas, 1996: 20; Penadés Martínez, 2012a: 35; García-Page, 2013: 252). En segundo lugar, el modismo presenta una serie de restricciones formales. Concretamente, los componentes léxicos de la locución son fijos e invariables, no pueden sustituirse por otros ni admiten el cambio de orden. Al mismo tiempo, la peculiaridad semántica de las locuciones presenta un alto grado de idiomaticidad. Es decir, en muchas ocasiones, el significado de una locución no equivale a la suma de los significados de sus elementos componentes. (ej.: como el perro y el gato). Por último, a diferencia de otras unidades léxicas compuestas como refranes (ej.: "la letra con sangre entra") y fórmulas diarias (ej.: “iUn momento!”), la locución es un elemento de la oración y no constituye enunciados completos.

En lo que se refiere a su clasificación, Corpas Pastor (1996: 93-110) distingue varios tipos de locuciones: locuciones nominales (ej.: media naranja), verbales (ej.: ponerse rojo), adjetivales (ej.: de por ahí), adverbiales (ej.: de arriba abajo), prepositivas (ej.: en función de), conjuntivas (ej.: asi que) y clausales (ej.: Subirsele [a alguien] la sangre a la cabeza). No obstante, actualmente, el concepto de locuciones clausales se ha ido desvaneciendo y, además, en el DRAE 2014, se las describe como locuciones verbales. En este artículo, para facilitar la estadística y el análisis posteriores, las consideramos también como tal. Asimismo, cabe señalar que esta clasificación se basa en la función gramatical que desempeñan las locuciones dentro de la oración. Desde este punto de vista, Casares (1992: 183) distingue entre locuciones significantes/de concepto (nominales, verbales, adjetivales y adverbiales) y locuciones conexivas/de enlace (prepositivas y conjuntivas). Dicho de otro modo, las primeras cuatro locuciones son unidades léxicas autosemánticas, en cambio, las dos últimas equivalen a un elemento de frase que desempeña el papel de conexión, pero no poseen significado léxico.

\section{La relevancia de las unidades fraseológicas en la enseñanza de ELE}

Cabría preguntarse por qué se deben enseñar las unidades fraseológicas, especialmente las locuciones. Respecto a su importancia, García Muruais expone las razones siguientes (1997: 364): 
1) Estas expresiones estereotipadas contribuyen a la economía discursiva.

2) El uso adecuado de las UUFF proporciona una mayor espontaneidad y fluidez al discurso oral, que resulta no sólo más vivo, sino también más auténtico.

3) Estas expresiones fraseológicas suponen cierto eufemismo como, por ejemplo, "Nuestro vecino no da su brazo a torcer". A través de esta expresión eufemística, el hablante transmite su opinión negativa de una forma indirecta y cortés.

4) El conocimiento de las expresiones fraseológicas resulta ser un útil decodificador para comprender lo que se quiere decir a partir de lo que se dice.

Además de que ayudan a aumentar la competencia comunicativa del hablante, las locuciones, destacan también por sus valores culturales. A propósito de esto, Núñez-Román (2015: 159) indica que numerosas expresiones fraseológicas incluyen referencias histórico-culturales como, por ejemplo, armar la de San Quintín, discusión bizantina, razón por la cual conocer el contexto cultural correspondiente es el único modo de descifrar su significado. Por otro lado, muchas expresiones fraseológicas tienen su origen en la literatura: no dejar títere con cabeza y poderoso caballero es don dinero, cuyos orígenes están en el Quijote de Cervantes y en Quevedo, respectivamente. Estos aspectos convierten a las unidades locucionales en punto de unión entre la lengua y la literatura. De modo que la inclusión de la fraseología en el aula de E/LE permite a los alumnos adentrarse en el universo cultural de la lengua meta y ofrecerles oportunidades de adquirir los conocimientos culturales en el proceso de estudiar las expresiones fraseológicas.

\section{Las principales propuestas didácticas de la adquisición de las locuciones}

A continuación, vamos a repasar y sintetizar las aportaciones principales en el ámbito de la adquisición de las locuciones desde tres perspectivas: la presentación de las locuciones, la descodificación y la conceptualización, y, por último, sus prácticas reales. Cabe señalar que estos tres aspectos corresponden a los tres pasos fraseodidácticos propuestos por Kühn (1992, apud Ettinger, 2008: 107), cuyo objetivo es reducir en la mayor medida posible las dificultades en el proceso de enseñanza-aprendizaje de las UUFF y ayudar a los estudiantes a reconocer, comprender, memorizar y reutilizar las locuciones aprendidas. 


\section{(1) La fase de presentación}

En esta primera fase, según Kühn (1992, apud Ettinger, 2008: 107), las locuciones deberán ser expuestas en un contexto debido a su carga cultural y uso sociopragmático. Asimismo, considerando su idiomaticidad y complejidad semánticas, contextualizarlas en un texto auténtico en vez de dejarlas aisladas constituye una manera efectiva para que el estudiante vea que las locuciones no se pueden explicar simplemente parafraseándolas, puesto que, en la mayoría de los casos, añaden un valor semántico diferente y están limitadas en su uso por cuestiones pragmáticas y situacionales (Szyndler, 2015: 208). Por lo tanto, el alumno tendría que identificar las unidades fraseológicas en un texto oral o escrito.

\section{(2) Explicación de significado y almacenamiento mental de las locuciones}

En lo que concierne a los modelos metodológicos de esta fase, se puede acudir a listas, tablas o inventarios, que sirven como instrumentos auxiliares (Forment Fernández, 1997: 341). Las listas han de contener todas las locuciones aparecidas anteriormente y su interpretación conceptual para que el estudiante comprenda qué quieren decir esas unidades estereotipadas. Asimismo, el objetivo principal de la didáctica de las locuciones consiste en enriquecer la capacidad comunicativa de los alumnos. En consecuencia, siguiendo la base del enfoque comunicativo, además de su explicación semántica, también hay que señalar en qué registro se debe utilizar la locución en cuestión, si se utiliza solo en la lengua oral o también puede ser usada en producciones escritas; cuáles son las características sociolingüísticas que presentan los hablantes que la emplean; qué tipo de información adicional proporciona al discurso en el que se intercalan (ej.: atenuación, intensificación, eufemismo, etc.) y cuáles son las condiciones de uso (Penadés Martínez, 1998: 126). Por otro lado, es recomendable asociar estas unidades idiomáticas con dibujos o ilustraciones representativos (Cooper, 1998: 263; Gómez Molina, 2004: 45). Tal y como propone Cooper (1998: 263), las locuciones pueden explotarse de forma efectiva recurriendo a dibujos o ilustraciones, puesto que estas expresiones estereotipadas suelen transmitir una imagen mental.

Aparte de eso, muchos estudiosos como Penadés Martínez (2004: 61) aboga por aplicar el enfoque contrastivo en la enseñanza-aprendizaje de las locuciones. Es decir, el profesor tiene que relacionar locuciones idiomáticas españolas con expresiones equivalentes o idénticas en la lengua materna del aprendiz y tener en cuenta la influencia de la L1 en la didáctica de las locuciones y como señala Penadés Martínez: 
La L1 desempeña un papel importante en la adquisición y uso de las unidades fraseológicas en la L2. En este sentido, se ha afirmado que los aprendices de una L2 no pueden ser vistos como un territorio fraseológicamente virgen, ya que tienen almacenadas un conjunto de ellas, propias de su lengua materna, las cuales, inevitablemente, juegan un papel, tanto positivo como negativo, en la adquisición de las de la L2 (2004: 61).

Por último, como sostiene Forment Fernández (1997: 341), la memorización es la única manera de manejar estas unidades idiomáticas y es importante elaborar mapas conceptuales para que los estudiantes las memoricen eficaz, científica y sistemáticamente. Partiendo de este objetivo, Penadés Martínez plantea proporcionar al estudiante locuciones sinónimas y antónimas de las que previamente le han sido presentadas (1999: 43). González Molina propone descubrir otras con estructura sintáctica similar (2004: 46). Por ejemplo, a partir de la locución adverbial $a$ ciegas, el profesor ha de añadir otras locuciones con la misma estructura: $a$ tontas y a locas, a secas, a escondidas, etc. Asimismo, siguiendo a la misma autora (González Molina, 2004: 46), agrupar las locuciones en virtud de su función también es un método importante, por ejemplo:

- modo (a ciegas, a sabiendas, con pelos y señales, a pedir de boca...)

- tiempo (cada dos por tres, en un santiamén, de vez en cuando...)

- lugar (al pie del cañón, boca arriba...)

También será de especial utilidad la presentación de las locuciones agrupadas por temas, palabras clave o por las metáforas (o metonimias) que hay tras ellas. En este sentido, Forment Fernández (1997: 342) formula un modelo metodológico concreto. La autora toma como ejemplo unos grupos de locuciones relacionadas con una parte del cuerpo humano y presentamos a continuación el más representativo:

\section{Locuciones formadas a partir del término cabeza ${ }^{3}$}

(a) Metáfora ontológica: LA CABEZA ES UN RECIPIENTE (no) caber [una idea] en la cabeza [a alguien] meter [una idea] en la cabeza [a alguien]

\footnotetext{
3 Este ejemplo ha sido extraído del artículo “La Didáctica de la Fraseología Ayer y Hoy: del Aprendizaje Memorístico al Agrupamiento en los Repertorios de Funciones Comunicativas" (Forment Fernández, 1997: 342-343).
} 
(b) Metonimia: LA CABEZA POR LA MENTE

calentarle [a alguien] la cabeza

romperse la cabeza

(c) Metonimia: LA CABEZA POR EL LIDERAZGO

a la cabeza de

en cabeza de

(d) Metáfora orientacional: LO BUENO ES ARRIBA Y LO MALO ES ABAJO

alzar la cabeza

bajar la cabeza

(e) Metonimia: LA CABEZA POR LA PERSONA

por cabeza

\section{(3) Uso y práctica}

El problema principal que presenta la didáctica de las locuciones es la memorización a largo plazo y la incapacidad de reconstrucción del significado por parte del alumno, razón por la cual es imprescindible aplicarlas en intercambios comunicativos reales para garantizar que los estudiantes tienen capacidad de reutilizarlas independientemente. En vista de eso, Penadés Martínez (2004: 61-63) plantea que el estudiante escriba una composición o cuente una historia en torno a su vida diaria utilizando las locuciones estudiadas de antemano $y$, además, imagine algunas situaciones en las que conversan con su interlocutor empleando algunos de los modismos estudiados. En estas actividades semilibres, el profesor tiene un rol importante de mediador y de guía para corregir los errores, dar la retroalimentación y proporcionar consejos de mejora.

\section{Objetivos, corpus y metodología de análisis}

El objetivo del presente trabajo es examinar si los manuales publicados en los últimos años han dado importancia a las locuciones y cuánto peso tienen en ellos. Concretamente, investigaremos numéricamente su frecuencia de aparición y examinaremos cualitativamente el grado de aplicación de las propuestas didácticas descritas en el apartado anterior. Con el fin de alcanzar estos objetivos, proponemos revisar y analizar los manuales del nivel B2, ya que, en el Marco Común Europeo de Referencia (el MCER), a propósito de la competencia sociolingüística, se precisa:

Desde el nivel B2, se ve que los usuarios son capaces de expresarse adecuadamente en una lengua que es sociolingüísticamente apropiada a 
las situaciones y personas implicadas y comienzan a adquirir [...] un grado mayor de control sobre el registro y los modismos" (Consejo de Europa, 2002: 118).

Los manuales que revisaremos son los siguientes:

(1) Abanico B2

Nivel: B2 del MCER

Editorial: Difusión

Fecha de publicación: 2011

(2) Aula Internacional 4 (Nueva Edición)

Nivel: B2 del MCER

Editorial: Difusión

Fecha de publicación: 2014
(3) Nuevo Sueña 3

Nivel: B2 del MCER

Editorial: Anaya ELE

Fecha de publicación: 2016

(4) Embarque 4

Nivel: B2 del MCER

Editorial: Edelsa, S.A.

Fecha de publicación: 2014

La selección de estos libros ha sido llevada a cabo considerando tres criterios básicos: primero, han sido editados siguiendo las directrices del MCER. En segundo lugar, debido a que uno de los objetivos del análisis es comprobar si se incorporan los estudios precedentes en la enseñanza de las locuciones en los manuales analizados, consideramos coherente analizar materiales publicados recientemente. Además, estos cuatro libros seleccionados se publican en tres distintas editoriales de ELE. Aunque Abanico B2 y Aula Internacional 4 (Nueva Edición) son dos manuales de la misma editorial, pertenecen a dos colecciones diferentes, en las que se reflejan distintas actitudes hacia las unidades locucionales. En cuanto a la metodología de análisis, primero vamos a realizar un análisis cuantitativo anotando el número y tipo de todas las locuciones aparecidas, en la forma que sea, en los manuales.

En segundo lugar, investigaremos cualitativamente cómo se enseñan las locuciones y qué tratamiento reciben. Las 10 preguntas siguientes suponen una síntesis de aquellos aspectos descritos en el marco teórico y nos sirven como criterios de análisis de cada manual.

1) ¿Hay un bloque especial para las locuciones? Si no ¿En qué parte(s) de la unidad didáctica se presentan las locuciones?

2) ¿Cómo se presentan las locuciones? ¿Las locuciones se ponen en un contexto o aparecen de forma aislada?

3) ¿Qué tipo de locuciones se trabajan? ¿cuál o cuáles tipos de ellas ocupan una mayor proporción?

4) ¿Las locuciones se corresponden a algún campo semántico concreto? ¿Las clasifican por las metáforas que hay tras 
ellas?

5) ¿Hay interpretaciones semánticas de todas las locuciones mencionadas anteriormente? ¿De qué forma o con qué ejercicios se explica su sentido?

6) Considerando la complejidad semántica de las locuciones y satisfaciendo la demanda expresiva del aprendiz, ¿se han señalado el registro, las condiciones de uso de las locuciones y su significado composicional?

7) Para facilitar la comprensión de las locuciones chay dibujos o ilustraciones? Aparte de eso, ¿se plantean otros modos de profundizar la comprensión de las locuciones presentadas?

8) ¿Se ha usado el enfoque contrastivo y se ha inspirado al alumno a vincular las locuciones españolas con las parecidas a su lengua materna?

9) ¿Se ha constituido el mapa conceptual? A partir de las locuciones presentadas, ¿se han añadido otras según su similitud semántica o formal?

10) ¿Hay práctica semilibre o libre con una o más de una actividad mediante la cual se consolida la capacidad de utilizar las locuciones de manera oral o escrita?

En el apartado que prosigue, discutiremos los resultados de estadística y análisis. Comentaremos los aspectos más relevantes para cada uno de los cuatro manuales.

\section{El tratamiento que reciben las locuciones en manuales de ELE}

\section{(1) El tratamiento que reciben las locuciones en Abanico B2}

En la tabla siguiente observamos el número de locuciones aparecidas en Abanico B2.

Cuadro 1. Resultado de estadística del número de locuciones en Abanico B2

\begin{tabular}{|c|c|c|c|c|c|c|}
\hline \multirow{2}{*}{$\begin{array}{c}\text { Abanico } \\
\text { B2 }\end{array}$} & $\begin{array}{c}\text { Locuciones } \\
\text { nominales }\end{array}$ & $\begin{array}{c}\text { Locuciones } \\
\text { verbales }\end{array}$ & $\begin{array}{c}\text { Locuciones } \\
\text { adjetivas }\end{array}$ & $\begin{array}{c}\text { Locuciones } \\
\text { adverbiales }\end{array}$ & $\begin{array}{c}\text { Locuciones } \\
\text { prepositivas }\end{array}$ & $\begin{array}{c}\text { Locuciones } \\
\text { conjuntivas }\end{array}$ \\
\cline { 2 - 6 } & 6 & 64 & 9 & 67 & 38 & 82 \\
\cline { 2 - 6 } & \multicolumn{3}{|c|}{ Locuciones significantes } & \multicolumn{2}{|c|}{120} \\
\hline \multicolumn{2}{|c|}{146} & Locuciones conexivas \\
\hline
\end{tabular}

En primer lugar, en Abanico B2 las locuciones aparecen siempre en contexto. Dicho esto, Ruiz Gurillo señaló que, en la mayoría de los 
manuales, toda la labor referente a la fraseología se encaminaba hacia una enumeración de estas unidades, fundamentalmente locuciones, sin aporte contextual sobre su uso (2000:2). Actualmente, este problema se ha mejorado en cierta medida en Abanico B2, ya que vemos al menos que todos los modismos presentados en este manual se han situado en un contexto concreto. Sin embargo, esos ejemplos y contextos adolecen de falta de falta de sentido práctico.

Además, a lo largo del libro, solo en los módulos 1, 3, 5, 11 existe un bloque dedicado a locuciones significantes, lo cual hace mayor hincapié en la habilidad para descifrar y reconocer las locuciones significantes, pero desatiende la capacidad para producirlas. Por su parte, la práctica semilibre con la cual se consolida la capacidad de reutilizarlas de modo oral solamente surge una vez a lo largo del libro (unidad 1, actividad 4c, p. 13). En cuanto al modo de organizar los modismos, las cuatro unidades arriba mencionadas los plantean según un tema determinado. Ponemos como ejemplo la unidad 5 (actividad 9, p. 82-83), en la que todos los modismos detallados tienen que ver con la comida, como darle [a alguien] las uvas, darse una piña [a alguien], etc., pero no se señalan cuestiones sobre la forma, la metáfora subyacente, el registro ni las condiciones de uso ni se incluye ninguna ilustración. Asimismo, se pone en práctica el enfoque contrastivo, animando al estudiante a relacionar las locuciones españolas con las correspondientes en su lengua materna (unidad 12, actividad $6 \mathrm{c}, \mathrm{p}$. 204). No obstante, este punto aparece también solamente una vez a lo largo de todo el libro. Aparte de eso, se debe señalar que Abanico 2 se limita a la enseñanza de las locuciones presentadas y no se añaden otras nuevas según la semejanza semántica ni formal.

Por último, también se les presta especial atención a las locuciones conexivas que funcionan como marcadores discursivos, por ejemplo, ya que, a causa de, etc. Estas expresiones no solo aparecen en casi todas las unidades, sino que también son muy variados los ejercicios orientados a su memorización y reutilización.

\section{(2) El tratamiento que reciben las locuciones en Aula Internacional 4}

He aquí el resultado de estadística del número de locuciones en Aula Internacional 4. 
Cuadro 2. Resultado de estadística del número de locuciones en Aula Internacional 4

\begin{tabular}{|l|c|c|c|c|c|c|}
\hline \multirow{2}{*}{$\begin{array}{l}\text { Aula } \\
\text { Internacional }\end{array}$} & $\begin{array}{c}\text { Locuciones } \\
\text { nominales }\end{array}$ & $\begin{array}{c}\text { Locuciones } \\
\text { verbales }\end{array}$ & $\begin{array}{c}\text { Locuciones } \\
\text { adjetivas }\end{array}$ & $\begin{array}{c}\text { Locuciones } \\
\text { adverbiales }\end{array}$ & $\begin{array}{c}\text { Locuciones } \\
\text { prepositivas }\end{array}$ & $\begin{array}{c}\text { Locuciones } \\
\text { conjuntivas }\end{array}$ \\
\cline { 2 - 7 } & 0 & 7 & 1 & 2 & 10 & 16 \\
\cline { 2 - 6 } & \multicolumn{3}{c|}{ Locuciones significantes } & \multicolumn{2}{c|}{ Locuciones conexivas } \\
\cline { 2 - 5 } & \multicolumn{3}{|c|}{10} & \multicolumn{2}{c}{26} \\
\hline
\end{tabular}

A través de estos datos puede verse que este manual no hace explícita la enseñanza de locuciones. Como muestra la tabla de arriba, es muy escaso el tratamiento de estas unidades fraseológicas, así que tampoco se fomenta el aprendizaje de dichas expresiones ni se pone en práctica ninguna teoría pedagógica sobre ellas. Aun así, en algunas secciones de expresiones escrita $u$ oral, se puede ver una serie de conectores, entre ellos se incluyen las locuciones conexivas, por ejemplo, en cambio, a causa de, debido $a$, etc., con la finalidad de que los estudiantes las manejen y aprendan a matizar con ellas lo que quieren expresar. No obstante, en cuanto a las locuciones significantes, observamos que Aula Internacional 4 aún las considera como un elemento prescindible y no las toma en consideración.

\section{(3) El tratamiento que reciben las locuciones en EMBARQUE 4}

En el cuadro siguiente exponemos el número de cada tipo de locuciones aparecidas en Embarque 4.

Cuadro 3. Resultado de estadística del número de locuciones en Embarque 4

\begin{tabular}{|c|c|c|c|c|c|c|}
\hline \multirow{2}{*}{$\begin{array}{c}\text { Embarque } \\
4\end{array}$} & $\begin{array}{c}\text { Locuciones } \\
\text { nominales }\end{array}$ & $\begin{array}{c}\text { Locuciones } \\
\text { verbales }\end{array}$ & $\begin{array}{c}\text { Locuciones } \\
\text { adjetivas }\end{array}$ & $\begin{array}{c}\text { Locuciones } \\
\text { adverbiales }\end{array}$ & $\begin{array}{c}\text { Locuciones } \\
\text { prepositivas }\end{array}$ & $\begin{array}{c}\text { Locuciones } \\
\text { conjuntivas }\end{array}$ \\
\cline { 2 - 6 } & 2 & 29 & 3 & 14 & 11 & 61 \\
\cline { 2 - 6 } & \multicolumn{3}{|c|}{ Locuciones significantes } & \multicolumn{2}{c|}{ Locuciones conexivas } \\
\cline { 2 - 6 } & 48 & \multicolumn{2}{|c}{72} \\
\hline
\end{tabular}

Cada unidad didáctica incluye locuciones significantes, pero se presentan de forma aislada y descontextualizada en una columna y con su significado en la columna de al lado, desordenadas, para que el estudiante los una. Asimismo, no se incluye una presentación de las unidades locucionales propiamente dichas, en la que se expliquen sus aspectos formales, de 
registro, sus condiciones de uso, etc. En cambio, debemos señalar que las locuciones significantes tratadas en cada unidad siguen una organización semántica, de acuerdo con lo sugerido por Forment Fernández (1997: 342). Así pues, en la unidad 1, por ejemplo, se encuentran todo tipo de modismos vinculados con la forma de habla: hablar por los codos, no tener pelos en la lengua, al grano, etc.

Además, cada lección trata las locuciones significantes de una manera bastante simple y similar y en todas se proponen los mismos tipos de ejercicios. El primero consiste en unir las locuciones con los significados que les correspondan. En el segundo ejercicio se debe rellenar un espacio tras la elección entre los modismos presentados antes y en el tercero, el estudiante debe ser capaz de reutilizar las unidades aprendidas de forma oral. En este sentido, ninguna actividad adopta una perspectiva de contraste con la lengua materna de los estudiantes ni incluye ilustraciones para visualizar la imagen subyacente. Excepto la monotonía del modelo de enseñanza, en comparación con la extensión enorme que ocupan otras secciones de este manual, como la gramática, el léxico, los conocimientos socioculturales, etc., el contenido de las locuciones significantes es realmente insuficiente y está muy poco desarrollado.

\section{(4) El tratamiento que reciben las locuciones en Nuevo Sueña 3}

Al igual que los tres primeros casos, primero, exponemos el número de cada tipo de locuciones encontradas en Nuevo Sueña 3.

Cuadro 4. Resultado de estadística del número de locuciones en Nuevo Sueña 3

\begin{tabular}{|c|c|c|c|c|c|c|}
\hline & $\begin{array}{c}\text { Locuciones } \\
\text { nominales }\end{array}$ & $\begin{array}{c}\text { Locuciones } \\
\text { verbales }\end{array}$ & $\begin{array}{c}\text { Locuciones } \\
\text { adjetivas }\end{array}$ & $\begin{array}{c}\text { Locuciones } \\
\text { adverbiales }\end{array}$ & $\begin{array}{c}\text { Locuciones } \\
\text { prepositivas }\end{array}$ & $\begin{array}{c}\text { Locuciones } \\
\text { conjuntivas }\end{array}$ \\
\cline { 2 - 7 } $\begin{array}{c}\text { Nuevo } \\
\text { Sueña }\end{array}$ & 7 & 26 & 6 & 77 & 19 & 71 \\
\cline { 2 - 6 } 3 & \multicolumn{3}{|c|}{ Locuciones significantes } & \multicolumn{2}{c|}{ Locuciones conexivas } \\
\cline { 2 - 6 } & \multicolumn{3}{|c|}{116} & \multicolumn{2}{c}{90} \\
\hline
\end{tabular}

Se observa que las locuciones significantes, clasificadas por compartir la misma palabra clave o la misma estructura, tienen presencia solamente en las unidades 1,4 y 5 , en las que hay una sección destinada a ellas, pero la mayoría se reduce prácticamente a las adverbiales y verbales, dejando en la sombra la enseñanza de las nominales y adjetivas. Además, es necesario destacar el hecho de que el libro presenta las locuciones significantes de forma aislada en un inventario, prescindiéndose de aportar la información sobre el registro, las condiciones de uso o su significado composicional. $E$ 
incluso, en la mayoría de los casos, no se hace referencia a los posibles contextos de utilización. A pesar de que en los ejercicios de la unidad 5 sí las pone en contexto, se trabajan en el nivel de la frase, y no en contextos superiores como discursos.

Asimismo, la mayor parte de los modismos presentados no abarcan ninguna explicación semántica y en este caso, el estudiante tiene que consultarla en el diccionario o deducirla a través del contexto dado en los ejercicios. En este sentido, cabe dejar constancia de que ninguna actividad del manual inspira al estudiante a relacionar las locuciones del español presentadas con las equivalentes en su lengua materna ni se incluye ningún dibujo para vislumbrar su imagen subyacente. A la vez, llamamos la atención sobre el hecho de que aproximadamente todos los ejercicios se centran en los aspectos semánticos, dejando a un lado la competencia de reutilización. Por otra parte, las locuciones conexivas que sirven para establecer relaciones semánticas entre distintas partes de un texto también ocupan un lugar importante en este manual. Estas expresiones aparecen en cada capítulo didáctico y al igual que Abanico B2 y Embarque 4, se ofrece un tipo variado de actividades para fomentar su retención.

\section{Conclusiones}

Los estudios en el ámbito de enseñanza-aprendizaje de locuciones de estos últimos años han influido positivamente en las nuevas propuestas para su adquisición en las clases de ELE, por tanto, en la mayoría de los manuales de ELE estudiados arriba, se ha realizado una explotación más profunda de las locuciones, pero también se han expuesto una serie de deficiencias comunes que se deberían subsanar.

Primero, en general, el tratamiento dado a las locuciones significantes es muy parco y su contenido está poco desarrollado. En este sentido, excepto en Embarque 4, los conocimientos de modismos no se han incorporado, como un elemento imprescindible, en todas las unidades didácticas sino solo en algunas de ellas. E incluso, una parte de los manuales actuales, representados por Aula Internacional 4, aún deja estas expresiones en un segundo plano sin hacer explícita su enseñanza.

En segundo lugar, la mayor parte de las actividades priorizan la práctica de aspectos relativos al significado, desatendiendo la capacidad de producirlas de forma oral o escrita. En este caso, como hemos mencionado anteriormente, el objetivo principal de la fraseodidáctica consiste en que el alumnado reutilice estas expresiones fluidamente y logre la máxima habilidad de expresión (Ettinger, 2008: 96). Paradojamente, en la mayoría de los casos no se relaciona el desarrollo del dominio de las locuciones con una mejora en el desarrollo de las destrezas comunicativas. 
Asimismo, es significativa la falta de información con respecto al registro, la condición de uso, la equivalencia idiomática en otra lengua, la metáfora que subyace en las locuciones, etc., y los alumnos tienen que buscar por sí mismos los aspectos arriba mencionados que se omiten en los materiales, razón por la cual concluimos que los manuales solo desempeñan un papel introductorio en el aprendizaje de los modismos y no pueden usarse como un material de apoyo eficiente.

Por último, las locuciones conexivas, por ejemplo, mientras que, en cambio, en cuanto a tienen una gran presencia en los manuales de ELE. A este tipo de locuciones se les otorga una gran importancia en todos los libros objeto de estudio, puesto que no solo surgen en casi todos los capítulos didácticos, sino que también son abundantes los ejercicios dedicados a su profundización y su memorización.

Finalmente, debido a la multiplicación de las investigaciones sobre este tema en los últimos años, han salido a la luz cuantiosos trabajos teóricos que exigen la existencia de criterios como la exhaustividad, la sistematicidad, la diversidad y la aplicabilidad en la enseñanza de este tipo de fraseología. Desafortunadamente, en los manuales de ELE más recientes, muchos de estos principios se han dejado a un lado.

\section{BibLIOgRAFía}

Alonso Cuenta, M. y Prieto Prieto, R. (2014): Embarque 4. Madrid: Edelsa S.A.

CASARES SÁNCHEZ, J. (1992): Introducción a la lexicografía moderna. Madrid: Consejo Superior de Investigaciones Científicas.

CONSEJO DE EUROPA (2001): Marco común europeo de referencia para el aprendizaje, la enseñanza y la evaluación de lenguas. Madrid: Anaya - Instituto Cervantes - MEC. Consultado en http://cvc.cervantes.es/ensenanza/biblioteca ele/marco/cvc mer.p $\underline{\mathrm{df}}$

COOPER, T. C. (1998): “Teaching Idioms". Foreign Language Annals, 31/2, pp. 255-266.

Corpas, J.; Garmendia, A.; SÁnChez, N. y SORIANo, C. (2014): Aula Internacional 4 Nueva Edición. Barcelona: Difusión.

CORPAS PASTOR, G. (1996): Manual de fraseología española. Madrid: Gredos. Chamorro, M. a D.; Lozano, G.; Martínez, P.; Muñoz, B.; Rosales, F. y Ruiz, J. P. (2011): Abanico B2. Barcelona: Difusión.

EtTINGER, S. (2008): "Alcances e límites da fraseodidáctica. Dez preguntas clave sobre o estado actual da investigación". Cadernos de Fraseoloxía Galega, 10, pp. 95-127. Consultado en: www.cirp.es/pub/docs/cfg/cfg10 06.pdf 
Fernández Prieto, M.a J. (2004): “La enseñanza de la fraseología. Evaluación de recursos y propuestas didácticas". En M. A. Castillo Carballo et al. (eds.), Las Gramáticas y los Diccionarios en la Enseñanza del Español como Segunda Lengua: Deseo y Realidad. Actas del XV Congreso Internacional de ASELE. Sevilla, Universidad de Sevilla, pp. 349-356.

García MuÑíals, M. a T. (1997): "Propuestas para la enseñanza de unidades fraseológicas en la clase de E/LE", en F. Moreno et al. (eds.), Actas del VIII Congreso Internacional de ASELE, Alcalá de Henares, Servicio de Publicaciones de la Universidad de Alcalá, pp. 363-369. Consultado

en: https://cvc.cervantes.es/ENSENANZA/biblioteca_ele/asele/pdf/08/ 08 0361.pdf

García-PAge, M. (2013): "En torno a la locución en el DRAE". Bulletin of Hispanic Studies, 17, pp. 251-260.

Gómez MolinA, J. R. (2004): "Las unidades léxicas en español”. La enseñanza de léxico en español como segunda lengua/lengua extranjera. Carabela, 56, 27-49. Consultado en: https://cvc.cervantes.es/ensenanza/biblioteca ele/carabela/pdf/56 $\angle 56$ 027.pdf

Álvarez, M. a Á.; Sanz, B.; De la Fuente, M.a V.; Giraldo, I.; Martín, F. y Torrens, M.a J. (2016): Nuevo Sueña 3. Madrid: Anaya ELE.

NúÑEZ-RoMÁN, F. (2015): "Enseñar fraseología: consideraciones sobre la fraseodidáctica del español". Didáctica.Lengua y Literatura, 27, pp. 153-166.

Penadés Martínez, I. (1998): "Materiales para la didáctica de las unidades fraseológicas: estado de la cuestión". Reale 9-10, pp. 125-146. Consultado en: https://core.ac.uk/download/pdf/58907781.pdf

PenAdÉs Martínez, I. (1999): La enseñanza de las unidades fraseológicas. Madrid: Arco/Libros.

Penadés Martínez, I. (2004): "La enseñanza de la fraseología en el aula de E/LE". Carabela, 56, pp. 51-67. Consultado en: https://cvc.cervantes.es/ensenanza/biblioteca ele/carabela/pdf/56 $\angle 56$ 051.pdf

Penadés Martínez, I. (2012a): Gramática y semántica de las locuciones. Alcalá de Henares: Servicio de Publicaciones de la Universidad de Alcalá.

Ruiz Gurillo, Leonor. (2000): “Un enfoque didáctico de la fraseología española para extranjeros", Especulo. Consultado en https://webs.ucm.es/info/especulo/ele/fraseolo.html

ReAl Academia EsPañola (2014): Diccionario de la lengua española (23. ${ }^{\mathrm{a}}$ ed.). Consultado en: https://dle.rae.es/

SzYNDLER, A. (2015): “La fraseología en el aula de E/LE: ¿un reto difícil de alcanzar? Una aproximación a la fraseodidáctica". Didáctica. Lengua 
y Literatura, 27, pp. 197-216. Consultado en:

https://core.ac.uk/download/pdf/38834073.pdf 